\title{
Are there differences in factors influencing access and continues use of assistive products for people with intellectual disabilities living in group homes?
}

\section{Fleur Heleen Boot, Malcolm MacLachlan \& John Dinsmore}

To cite this article: Fleur Heleen Boot, Malcolm MacLachlan \& John Dinsmore (2019): Are there differences in factors influencing access and continues use of assistive products for people with intellectual disabilities living in group homes?, Disability and Rehabilitation: Assistive Technology, DOI: $\underline{10.1080 / 17483107.2018 .1550116}$

To link to this article: https://doi.org/10.1080/17483107.2018.1550116

\section{曲 Published online: 28 Jan 2019.}

\section{Submit your article to this journal ¿}

山 Article views: 14

View Crossmark data ¿ 


\title{
Are there differences in factors influencing access and continues use of assistive products for people with intellectual disabilities living in group homes?
}

\author{
Fleur Heleen Boot ${ }^{a, b}$, Malcolm MacLachlan ${ }^{a, c, d}$ and John Dinsmore ${ }^{e}$ \\ ${ }^{a}$ Assisting Living \& Learning (ALL) Institute, Maynooth University, Maynooth, Ireland; ${ }^{b}$ Centre for Global Health, Trinity College Dublin, Dublin,

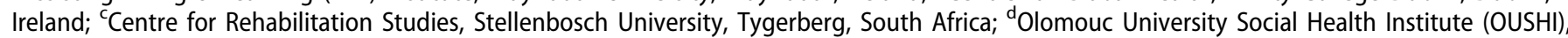 \\ Palacky University, Olomouc, Czech Republic; ${ }^{\mathrm{e} C e n t r e}$ for Practice and Healthcare Innovation, Trinity College Dublin, Dublin, Ireland
}

\begin{abstract}
Background: The World Health Organization has launched a program to promote Global Cooperation on Assistive Technology (GATE) to implement those parts of the United Nations Convention on the Rights of Persons with Disabilities (UNCRPD) referring to assistive technology and products. A particular vulnerable group deeply affected by health inequity affecting access to assistive products are people with intellectual disabilities.

Objective: The objective of this study is to understand the barriers and facilitators to effectively access and continuously use essential assistive products for people with intellectual disabilities.

Materials and methods: Face-to-face interviews with 15 adults with a mild to profound intellectual disability and 15 providers of assistive products were conducted to gain insights about current use, needs, knowledge, awareness, access, customization, funding, follow-up, social inclusion, stigma and policies around assistive products and intellectual disability. The technique of constant comparative analysis was used to analyze the data.

Results and conclusion: An overview of factors is presented that fit within seven domains for access and eight domains for continuous use of assistive products for people with intellectual disabilities. It illustrates that access and continuous use are influenced by different barriers and facilitators. These different influences should be taken into account in country policies and frameworks that seek to implement the UNCRPD through assistive technology.
\end{abstract}

ARTICLE HISTORY

Received 26 July 2018

Revised 9 November 2018

Accepted 15 November 2018

\section{KEYWORDS}

Intellectual disability; assistive technology; assistive products; access; health inequity

\section{Introduction}

Assistive products (AP) can improve a person's daily functioning, independence and inclusion, and prevent impairments and secondary health conditions [1]. The importance of AP has been acknowledged at international level. The Sustainable Development Goals (SDG's) envision an inclusive world, where no one is left behind, and each of the SDG's can be facilitated by the use of AP [2]. The United Nations Convention on the Rights for People with Disabilities (UNCRPD) stipulates access to AP as essential. Following the UNCRPD, the World Health Organization (WHO) launched a program called Global Cooperation on Assistive Technology (GATE) to support countries in developing national policy programs, training packages for professionals, and service delivery models around assistive technology, all to improve access and use of AP for those in need [1]. The GATE program has developed a priority Assistive Product List (APL). The APL represents a minimum list and includes 50 priority AP, selected on the basis of widespread need and impact on a person's life [3]. The 50 priority AP are both low- and high-tech AP in the domains of vision, hearing, mobility, communication, cognition, environment and personal care. Examples are spectacles, hearing aids, (motorized) wheelchairs, communication software, pill organizers, portable ramps and incontinence products.

People with intellectual disabilities (ID) are a specific group that could greatly benefit from AP [4-7]. ID is defined by the American Association on Intellectual and Developmental Disabilities (AAIDD), the Diagnostic and Statistical Manual of Mental Disorders (DSM-V) and the International Classifications of Diseases (ICD-10, mental retardation) as an IQ below 70, manifested during the developmental period (<18years of age), with impairments in adaptive 
functioning, such as communication skills, social skills, personal independence, school or work functioning [8-10]. AP can support the cognitive limitations people with ID encounter and may enhance independence, education, employment, social activities, community living and inclusion [4]. In addition, people with ID have a higher prevalence of comorbidities compared to the general population that could be better managed with AP, such as sensory impairments, speech and language impairments, and motor disabilities [11-13]. With the current ageing trend, the prevalence of older people with ID is likely to increase along with the demand for access to AP $[6,14]$.

However, people with ID use fewer AP compared to other populations in need; which negatively influences the health inequalities already present for people with ID [11,15-17]. The health needs of people with ID are often unrecognized and unmet, and new diagnoses are frequently missed $[11,18]$. People with ID are still generally regarded as belonging to a devalued and stigmatized group, and are a particular vulnerable group deeply affected by health inequity affecting access to healthcare and AP $[11,19]$. Public health and social care services in countries like Ireland fund a range of services for people with ID, but people still face difficulties in accessing those services, including AP [20]. Access to high-quality affordable AP for people with ID is becoming a high priority for countries' future policy programs. The WHO GATE program encourages countries to develop their own APL according to needs and context, and to commit to implementation of the APL. By ratifying the UNCRPD, countries including Ireland commit to make changes in current inequities regarding access to and use of AP for people with ID.

In order to improve access to AP for people with ID aspired by the GATE program, we need to sufficiently consider the barriers that people with ID currently experience regarding access to AP, and identify how effective access can be promoted. The International Classification of Functioning, Disability and Health (ICF) addresses barriers as factors in a person's environment which limit functioning and create disability, while facilitators are factors that improve functioning and reduce disability [21]. A recent systematic review by Boot et al. identified the currently available scientific evidence on barriers and facilitators for people with ID to access AP [22]. The three main barriers found in literature were related to $A P$ costs, lack of awareness about $A P$, and inadequate assessments for people with ID. The three main potential facilitators found in the literature focus on (1) increasing knowledge and awareness around the benefits of AP use for people with ID (2) increasing funding opportunities for persons with ID to acquire $A P$, and (3) embedding AP within the daily lives of people with ID, e.g. making AP part of their support plan. However, this review also highlighted a lack of research to sufficiently understand the barriers for people with ID to access AP in differently resourced settings and stipulated the need for future research activities to include people with ID themselves as experience experts as part of an AP co-design and development approach.

Following access to AP, the importance of continuous use of AP by people with ID should not be overlooked. After AP is obtained there is a high rate of nonuse or product abandonment [17]. For AP users in general, the overall abandonment rate has been $\sim 30 \%$ [23]. Possible reasons for abandonment may be due to the assignment of inappropriate AP, or failure to meet user needs and expectations [24,25]. For people with ID intellectual limitations may influence their skills to use AP independently and consequently determine the potential dependence on others for support $[17,26,27]$. However, little is known about the specific abandonment rate for people with ID and which factors influence their abandonment.

Access to and the use of AP for people with ID is still a neglected area in research and practice. Without a concerted and systematic approach to consider the challenges that ID presents, for the users, caregivers, and providers of AP, profound inequities in health, in life opportunities, and therefore in the quality of life for people with ID will persist. The objective of this study was to understand the barriers and facilitators to effectively access and continuously use essential AP for people with ID.

\section{Materials and methods}

\section{Study design}

This study consisted of a qualitative research design using semistructured face-to-face interviews with a phenomenology approach. Relatively few people with ID are able to read and write and to fill in written questionnaires. Therefore, the most appropriate method to gain personal views from people with ID is interviewing. The interviews took place from May 2017 to October 2017. Ethical approval for this study was granted by the Research Approvals Subcommittee of the Daughters of Charity Service Ethics Committee, Ireland (004/2017). This study is part of the large crosssectional study Global Access to Assistive Technology for People with Intellectual Disabilities (GATE-ID), for which ethical approval was obtained from the Health Policy \& Management/Centre for Global Health Research Ethics Committee, Trinity College Dublin, Ireland (04/2017/01) and Maynooth University Research Ethics Committee, Ireland (SRESC-2017-053). The study adhered to the Declaration of Helsinki for research involving human subjects.

\section{Participants}

The participants were divided into two main groups; (1) adults with ID, and (2) providers of AP. Purposive sampling was used to recruit participants. Participants of group (1) adults with ID were approached through an Irish ID service provider with locations in the Dublin and Limerick area. The managers of the group homes were informed about the study and asked to select 15 adults with ID willing to participate in an interview, either users or nonusers of AP. Preference was given to select a range of individuals with different ages and levels of ID. The information leaflet and consent form were adjusted to the cognitive level of the participants i.e., easy to read, larger font size, short amount of words per row and the use of symbols. If the participant was not able to give informed consent, his or her legal representative gave informed consent. After informed consent, information on age, gender, care setting, level of ID, cause of ID, medical history, and indication or reason for having the AP was gathered. This information was provided by the care staff or nurses of the ID service provider. Level of ID was categorized into borderline, mild, moderate, severe, and profound ID, according to ICD-10 classification. Depending on the cognitive level and communication possibilities of the participant, the interviews were conducted with the persons with ID themselves and/or their care staff. Participants of group (2) Providers of AP consisted of prescribers of AP (health professionals working with people with ID), suppliers or retailers of AP, managers of public funding authorities and managers of disabled person's organizations. Eight participants for group 2 were approached through Irish ID service providers and snowball sampling was used to then achieve the target of 15 . The number of 15 participants per group was expected to be sufficient to achieve saturation of the data from interviews [28]. Achieving data saturation 
TABLE 1. Participants characteristics.

\begin{tabular}{lc}
\hline Characteristics & People with ID $(n=15)$ \\
\hline Age (mean) & 54 years \\
Female / Male & $15 / 0$ \\
Level of ID & \\
Mild-moderate & 9 \\
Severe-profound & 6 \\
Aetiology & \\
Cerebral palsy & 3 \\
Meningitis / encephalitis & 2 \\
Down syndrome & 1 \\
Prader Willi syndrome & 1 \\
Unknown & 8 \\
Care setting & \\
Community group home & 10 \\
Centralized setting & 5 \\
Independent & 0 \\
With family & 0 \\
Urban / Rural & $15 / 0$ \\
Characteristics & $(n=15)$ \\
Age (mean) & 41 years \\
Female / Male & $13 / 2$ \\
Work field & \\
S\&L therapist & 3 \\
OT & 2 \\
Physio & 2 \\
MD & 1 \\
Supplier of AP & 2 \\
Manager public funding authority & 4 \\
Manager DPO & 1 \\
Urban / Rural & $14 / 1$ \\
\hline S\&L: Speech and lavider & \\
\hline
\end{tabular}

S\&L: Speech and language; OT: Occupational therapist; MD: Medical doctor; AP: Assistive products; DPO: Disabled persons' organization.

will provide a comprehensive account for the specific groups interviewed in that resource setting. Data saturation was achieved when no new information from further interviews was obtained. If the number of interviews did not achieve saturation of data, additional participants were selected.

\section{Interviews}

The first author developed the interview guide based on key themes present in the literature supplemented by expert opinion, which included a professional with expertise in policy, organization and systems perspectives regarding people with disabilities, and a professional with expertise in AP training services. The interview guide was vetted with colleagues at the Centre for Global Health, Trinity College Dublin, to solicit feedback on the scope, clarity, and flow of questions and, where appropriate, the interview guide was refined. Collaboration with two research assistants from Inclusive Research Network Ireland (both with a mild ID) took place to develop a more user-friendly interview guide for participants of group 1. The Inclusive Research Network Ireland initiated in 2008 as a partnership between people with ID and academic researchers to promote and develop person-centered research programs [29]. The interview guide was then piloted with an adult with mild ID to evaluate framing and clarity of questions. The semi-structured interview guide focused on AP current use, needs, knowledge, awareness, access, customization, funding, follow-up, social inclusion, stigma and policies. A different interview guide for each participant group was used. A copy of the interview guides is provided as electronic supplements 1 and 2. Interviews were conducted at a time and location suitable to the participant. The researcher explained the purpose of the interview and asked permission to use an audio recorder. At the start of each interview, AP were defined using a booklet containing images of the $50 \mathrm{AP}$ from the APL to highlight the variety in
AP. Care staff were present if needed, to give support to the participant or clarify the respondents' pronunciation. The questions were adjusted to the level of ID and the care staff aided participants in understanding questions they found challenging. Interviews started with an open-ended question. The open dialog was promoted through the interview. Following participants' open responses, topics were concluded primarily with closedended questions.

\section{Analysis}

The researcher transcribed verbatim all data in full from recordings of the interviews. The technique of constant comparison analysis, as described by Elliott and Timulak [30], was used to analyze the data. Accordingly, the data was distributed into meaning units - units by which the analysis was conducted. Meaning units are segments of the data that even if interpreted out of context would communicate adequate information to convey a piece of meaning to the reader [30]. The meaning units were divided into meaning units for access to AP and meaning units for continuous use of AP. Broad headings, or domains, for organizing participants' responses were identified. These domains were developed predominantly during data coding. Several organizing frameworks were flexibly developed and tested until they were deemed to appropriately fit the data. The meaning units were subsequently coded or categorized. As such, categories emerged from the meanings in the meaning units. Formulation of categories is an interpretive and interactive process whereby the researcher labels categories similar to the actual language of participants, while also applying their own understanding and knowledge of previous theory and study findings [30]. The categories were labeled as "barrier" , "facilitator" , or as "both" if it could act as either a barrier or facilitator. Barriers were defined as factors that limit or inhibit persons with ID to access or use AP. Facilitators were defined as factors that potentially facilitate, encourage or enable persons with ID to access or use the AP they need. All categories that were mentioned by three or more participants were presented. The categories that were only mentioned by one or two participants were included in the results section if the meaning unit was of such relevance and importance to the domain, judged by the first author.

\section{Results}

\section{Participants characteristics}

In total 30 participants were interviewed. Table 1 presents the participants' characteristic of both group (1) Adults with ID $(n=15)$ and group (2) Providers of AP $(n=15)$. Due to the history of the ID service provider where participants of group (1) were recruited, all adults with ID were females. Thirteen adults with ID were accompanied by their carer during the interview. Six of these adults were non-verbal and/or had a severe-profound ID in which case the carer answered all the questions for them. Three adults with ID were comfortable and able to do the interview completely independent.

\section{Assistive products in use}

Table 2 presents the current use of AP by the participants from group (1) Adults with ID. The participants used eight AP on average, ranging from 3-13. Products for environment and self-care, and mobility were most often used. None of the participants were using AP to support hearing. 
TABLE 2. Current AP in use by participants of group (1) adults with ID.

\begin{tabular}{|c|c|c|c|c|c|c|c|c|c|}
\hline Code & Total no. of AP in use & APL & Non-APL & Hearing & Vision & Communication & Mobility & Cognition & Environment and self care \\
\hline INT_ID_IRL_001 & 5 & 4 & 1 & 0 & 1 & 2 & 1 & 0 & 1 \\
\hline INT_ID_IRL_002 & 3 & 3 & 0 & 0 & 1 & 1 & 1 & 0 & 0 \\
\hline INT_ID_IRL_003 & 9 & 8 & 1 & 0 & 2 & 2 & 2 & 0 & 3 \\
\hline INT_ID_IRL_004 & 13 & 10 & 3 & 0 & 1 & 2 & 4 & 0 & 6 \\
\hline INT_ID_IRL_005 & 11 & 10 & 1 & 0 & 1 & 2 & 3 & 0 & 5 \\
\hline INT_ID_IRL_006 & 10 & 10 & 0 & 0 & 1 & 0 & 3 & 0 & 6 \\
\hline INT_ID_IRL_007 & 7 & 6 & 1 & 0 & 0 & 2 & 1 & 0 & 4 \\
\hline INT_ID_IRL_008 & 8 & 7 & 1 & 0 & 1 & 1 & 3 & 0 & 3 \\
\hline INT_ID_IRL_009 & 7 & 6 & 1 & 0 & 0 & 1 & 1 & 0 & 5 \\
\hline INT_ID_IRL_010 & 5 & 3 & 2 & 0 & 0 & 0 & 1 & 0 & 4 \\
\hline INT_ID_IRL_011 & 7 & 3 & 4 & 0 & 0 & 0 & 2 & 0 & 5 \\
\hline INT_ID_IRL_012 & 10 & 8 & 2 & 0 & 0 & 1 & 4 & 0 & 5 \\
\hline INT_ID_IRL_013 & 7 & 6 & 1 & 0 & 0 & 1 & 2 & 0 & 4 \\
\hline INT_ID_IRL_014 & 5 & 3 & 2 & 0 & 0 & 1 & 2 & 0 & 2 \\
\hline INT_ID_IRL_015 & 6 & 2 & 4 & 0 & 0 & 1 & 2 & 0 & 3 \\
\hline TOTAL & 113 & 89 & 24 & 0 & 8 & 17 & 32 & 0 & 56 \\
\hline Average & 8 & 6 & 2 & 0 & 1 & 1 & 2 & 0 & 4 \\
\hline
\end{tabular}

\section{Qualitative analysis}

Domains are illustrated schematically in Figure 1(a,b); these domains are not ordered in terms of importance nor do they imply any hierarchy. Figure $1(a, b)$ illustrates if a category was mentioned by 10 or more participants ("Bold" font), by three to nine participants ("Regular" font), or only by one or two participants ("Italic" font). The categories were labelled as "barrier", "facilitator", or as "both" if it could act as either a barrier or facilitator. Each quote provided below ends with the code of the participant in parentheses. Codes consist of the letters "ID" for group (1) Adults with ID and "PRO" for group (2) Providers of AP.

\section{Barriers and facilitators for access to AP}

Meaning units were organized as categories within 7 domains: AP availability; assessment; attitudinal \& social context; financial; knowledge and awareness; organizational and systemic structural; and physical access \& transport. The number of categories per domain varied from two to six, see Figure 1(a).

AP Availability. Participants indicated that the provider of AP was available within close commuting distance to the user. For example, one participant $(P)$ stated following the question of the researcher (R): "R: And do you remember who gave you the glasses? $P$ : The optician in town". (INT_ID_IRL_004). Participants reported a wide range of AP relevant to their needs available within their country. One participant compared this aspect with resources available in other lower income jurisdictions: " $P$ : We're very lucky. We have a range of products. I mean we've had OT's come from, Zimbabwe, from Kenya and they would have an ordinary chair, cut $a$ hole in the chair, and put a basin in and that's a commode. And we have dozens of commodes to choose from". (INT_PRO_IRL_011). Another participant did worry about the lack of national AP production, and stated: "P: When we were talking about Brexit that was one of the things I was listing as an issue for us because we don't make any equipment ourselves... A lot comes through the UK so we may have to source other markets as that moves forward". (INT_PRO_IRL_014).

Assessment. Four categories were of influence regarding correct and useful assessment for people with ID, to identify which AP is needed for that person. User involvement within trial and assessment of AP was seen as a facilitator to ensure the correct AP for users could be accessed: "P: Where someone is able to help to choose themselves we would certainly involve them. So if someone is going through a trial process, where they're trialing out a number of different devices, I might do a quick survey with them afterwards to see what they liked about it and what they didn't like. And I would go back to the suppliers with that then and say; she really liked the screen size, she didn't like that it took so long to get to the symbol or whatever it is, so that they're able to then say; oh well actually I have this other device that's much more suitable then". (INT_PRO_IRL_002). Participants recommended that professionals assess AP in an environment that is suitable to the user to ensure the accuracy of assessment. In addition, a proactive approach from the professionals to assessing specific health problems and AP needs for people with ID was viewed as key to facilitating appropriate AP deployment to the user. However, this was not often reported: " $R$ : And is her sight or her hearing ever been tested or assessed? P: Her sight, not in the last few years, I've been here 4 years and sight has never been tested, nor hearing. But, you know, when you're talking to $A$ it doesn't seem to appear she has any problems with that". (INT_ID_IRL_007). Carer involvement during assessment was seen as essential to access the correct AP, since the carer has insight into which AP may best suit the user and they would be able to recognize the user's responses during assessment. In some cases the providers of AP did not include the carer.

Attitudinal and social context. Access to AP for people with ID also depended on carers being familiar to new AP and carers being open to new AP: "P: some families would say oh well this is brilliant and they would be totally up for it. But you do get some families that say, oh no, what would you be needing that for? Or like they can use a normal knife. And you're kind of going, well I've seen them and this is really going to help". (INT_PRO_IRL_001). Several participants mentioned that AP for people with ID was still mainly focused on comfort, caring and medical needs, with less emphasis on development, inclusion or participation in society: "P: a wheelchair is a P1 whereas a communication device is at the bottom of the list, it is considered a P3. R: Less urgent. P: It would be deemed less urgent according to this rating scale". (INT_PRO_IRL_010). Mainstream AP seemed to positively influence the attitudes of people towards AP: "P: I think especially with, now with iPads and things like that, that it's a very accepted method of communication". (INT_PRO_IRL_002). However, due to the current paternalistic attitudes of carers and professionals to people with ID, access to AP could be prohibited, as stated by one of the participants: "P: I think people have the same rights no matter what their disability is. I don't think that is understood within the system, there is very, very paternalistic, we have a very paternalistic attitude to people with intellectual disabilities. I was in a service recently and 
(a)

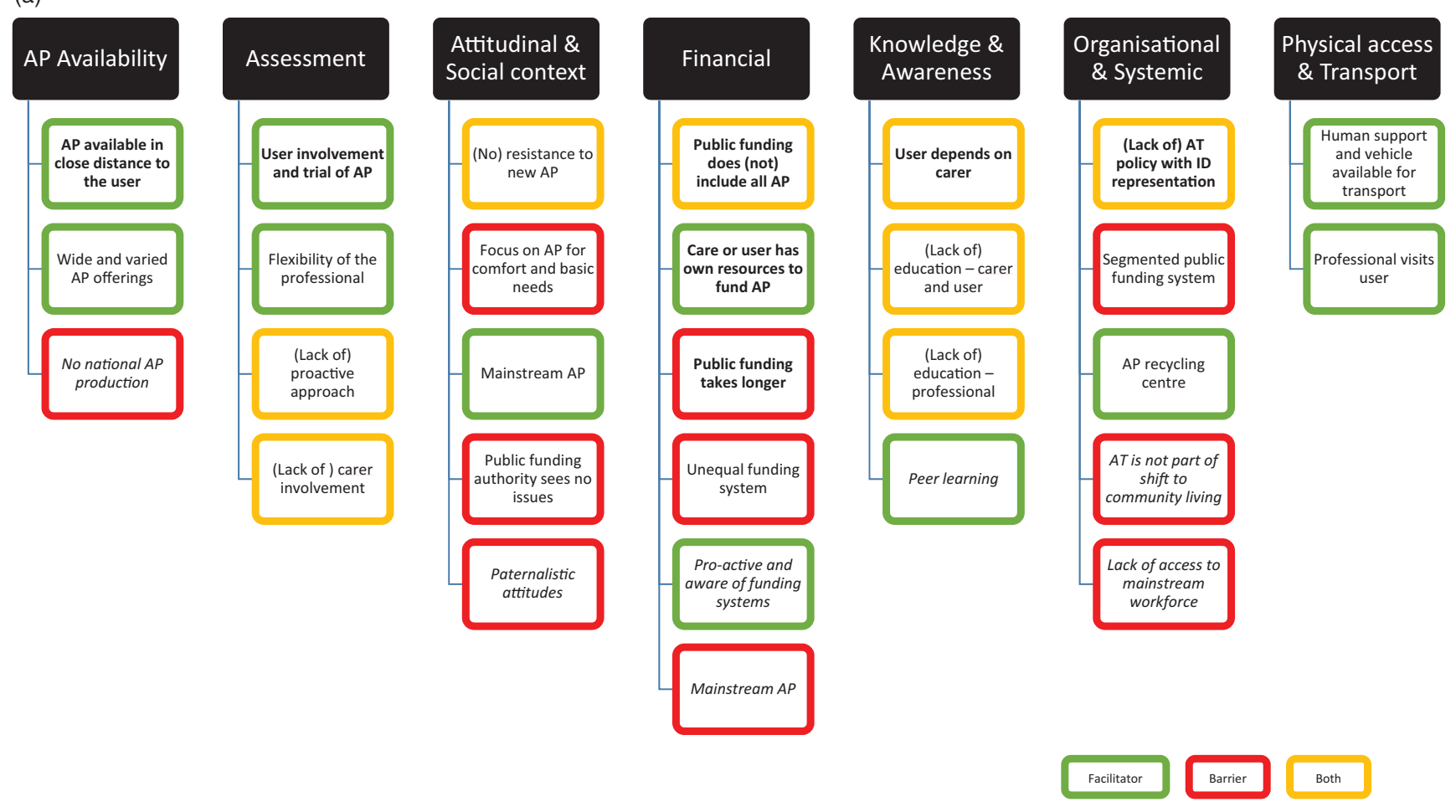

(b)

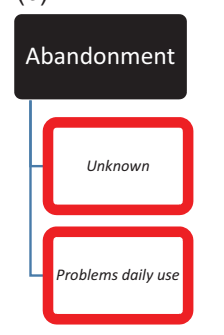

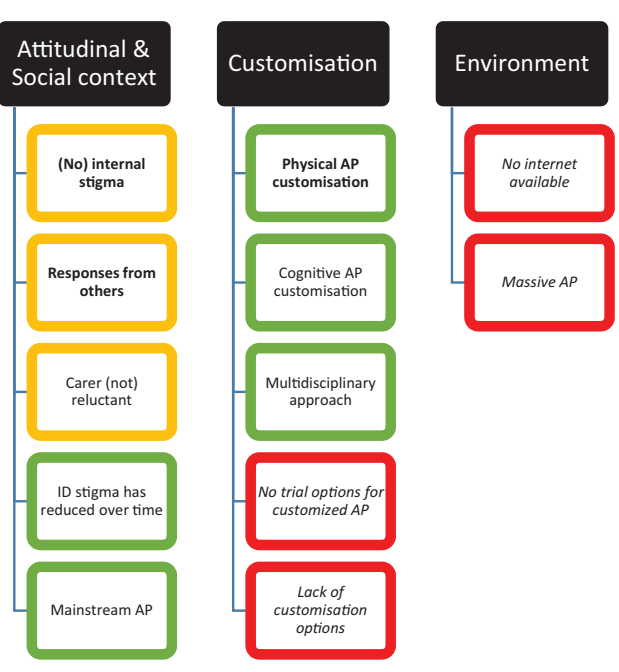

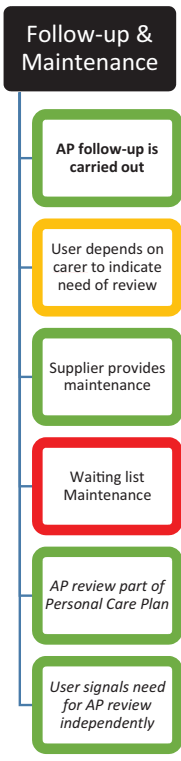

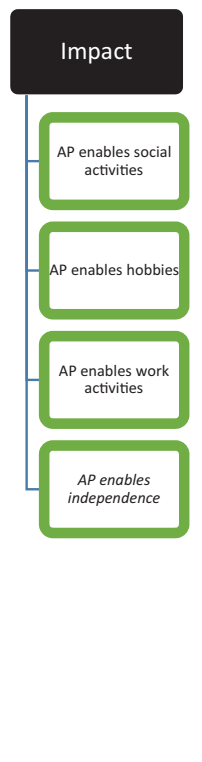

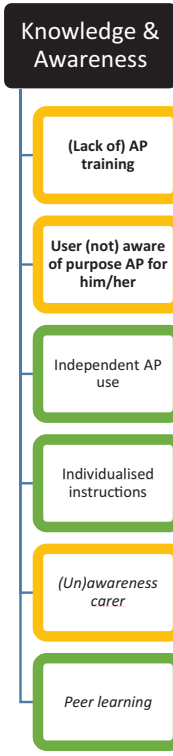

\section{Support}

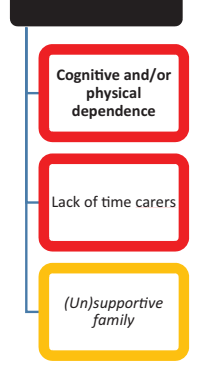

Facilitator

Barrie

Both

Figure 1. (a) Domains and categories for factors influencing access to AP for people with ID. (b) Domains and categories for factors influencing the continuous use of AP for people with ID. A category in Bold font represents $\geq 10$ participants, in Regular font represents 3-9 participants and in Italic font represents 1-2 participants.

the instructor in the service was talking about how they more or less take people's mobile phones off them in the morning time because they would be texting during the day". (INT_PRO_IRL_015).

Financial. Public funding was mainly available for AP that was seen as a basic need and comfort, with a focus on AP for medical issues. For example, one participant explained that her glasses were publicly funded. However, other AP listed on the APL, was not available through public funding even if the importance to a user was noticed by the public funding authority: "P: Because the OT's justification for it was really good but it just didn't fit in with our, I couldn't see the functional aspect of it from our disciplines point of view but I could see how useful it would be to the family. But just from a funding thing". (INT_PRO_IRL_007). Access to AP was easier and quicker if people had their own financial resources to acquire the AP they needed. Access to AP generally took longer if public funding was used due to waiting lists, with up to 18 months waiting time according to one participant. Another 
participant explained: " $P$ : we are trying to get her a chair through HSE [public funding authority]. But it takes a long time! Like we started already before, and it is still, we don't know. Because she has no money, and we need to go through HSE. And we still don't have the chair to be honest with you". (INT_ID_IRL_012). Strict eligibility criteria applied for individuals seeking assistance for the provision of AP via publicly funded medical or long-term illness schemes and not all people with ID fitted the criteria, especially individuals with a mild to moderate ID. For those people with ID who did fit the criteria and applied via publicly funding schemes for AP support, had to face another barrier; their applications were grouped with applications from other populations in need of AP and were often assessed as being less urgent which increased waiting time: " $P$ : The applications for people with intellectual disabilities were often treated less urgently. They were often treated less urgently because they share the same pool of money as people who are coming out of hospital quickly or people with physical disabilities who were at risk of spinal deformity". (INT_PRO_IRL_007). People with ID also depended on the approach of the health professional to support access to funding. However many participants reported interacting with a high number of different health professionals throughout their lifetime, as such consistency to AP support varied. The mainstream AP market could benefit access to AP for people with ID, as stated by one of the participants: "P: if you can show you can bring industry to Ireland then you will gain more traction because you have to mainstream it. I think mainstream tech companies, employment, other departments will get it before we change the policy that delivers the stuff on the ground". (INT_PRO_IRL_015)

Knowledge and awareness. People with ID often depend on a carer to indicate the need for AP and to make the appointment for assessment. And thus, they depend on others to have access to AP. However, carers reported a lack of knowledge on available AP and the benefits of AP use for people with ID: "P: There is probably so much out there that we probably don't know about. It's why I definitely think that staff needs to be kept updated of what's out there". (INT_ID_IRL_009). Professionals often felt responsible to educate the user and carer about available AP. To be capable of providing that, professionals stated that they needed to be kept up-to-date on new, more appropriate AP that was available in their field. However the professionals often felt there was a lack of (appropriate) training for them specific to the area of ID: "P: it is often the case that, you know, when we go to different training days and things I find are really, really interesting and I come back with a lot of information. But then actually putting that into practice is difficult, because often the people in mind in these training days are people without ID. So obviously you are talking about a very different client group there. Certainly it would be great to have more information and more devices available, or adaptations available". (INT_PRO_IRL_002). Increasing awareness among people with ID about available AP could influence their motivation to access and use AP. One method mentioned to increase awareness was peer learning: " $P$ : they would almost want to see examples of other people with similar disabilities using some technology which they could say, gosh I didn't realize I could do that and if ever I was able to do that then ... And I think until people can see that other people are using assistive technology it is hard to understand how because you can't imagine..." (INT_PRO_IRL_014)

Organizational and systemic. Participants were hoping that the UNCRPD would make the State take more responsibility to improve current policies and funding for AP specific to ID. They felt that the gap between policy and implementation was enormous and that there was a need for improving guidance on how to obtain access to tailored AP relevant to individual needs. Participants worried about a new national AP list which was going to be rolled out that did not include options for specialized or personalized AP. In addition, participants mentioned the importance of the right people being involved in policy developments: "P: so they have about four different committees looking at different aspects of it [National AT program]. But the OTs that I know that are on it are not involved in disability services, they are the OTs that are seeing the people with strokes in the community. So I think a national look at it would be good but I do think that individual groups have to be represented strongly". (INT_PRO_IRL_007). The current system for public funding varies in Ireland depending on geographical location. This has resulted in different price structures for acquiring AP and waiting times for assessment and receipt of AP: "P: That was within our area, that long wait time. In other areas in Ireland they might have a bigger budget or smaller population so things might get funded more quickly". (INT_PRO_IRL_007). Initiatives like an AP recycling or lending center were very much encouraged to decrease costs. Participants underlined the importance of the role of AP with the current shift towards community living for people with ID, however purchasing models for AP are not integrated into the national budget to reflect this shift: " $P$ : They are all into congregated setting and moving into the community. But how can you move when you don't have the right equipment. The right wheelchair, the right facility. It all sounds great on paper, but if the funding is not there". (INT_ID_IRL_009). One participant indicated the importance of access to the mainstream workforce for people with ID to have access to AP: "P: They are doing the cute [minor] jobs and that they are not doing anything of actual value, they are not part of the mainstream workforce. And the mainstream workforce is working remotely, is extremely mobile, has access to the technology and has technological skills". (INT_PRO_IRL_015)

Physical access and transport. Transport was not stated as a barrier for people with ID to access AP in this context. Participants reported that carers supported their transport needs with suitable vehicles always available to visit AP providers or professionals. For example: "R: And do you remember how you got there? Could you walk? P: I didn't walk... P: Bus. R: With the bus. And was it the public transport, or the bus from the [service provider]? P: From here". (INT_ID_IRL_005). And often the users did not even have to travel, because the AP provider or professional would visit the user at their home or daycare center for assessment.

\section{Barriers and facilitators for continuous use of AP}

Meaning units were organized as categories within eight domains: Abandonment; Attitudinal \& Social context; Customization; Environment; Follow-up and Maintenance; Impact; Knowledge and Awareness; and Support. The number of categories per domain varied from two to six, see Figure 1(b).

Abandonment. AP abandonment was not common for users living at ID service organizations. If it did happen, the main factors of influence were actually unknown, or there was a practical problem with using the AP: "R: You also told me you have the hearing aids, you have them but you don't use them anymore? P: Not really. It comes off. R: It falls off? But if you have them in, do you hear better? P: I could yeah... ... I can hear you now, you're near me. If people, I used hearing aids, if people are far away I can't hear them calling". (INT_ID_IRL_003) 
Attitudinal and social context. Most of the users didn't feel ashamed using their AP and liked their AP, especially when they noticed the benefit of the AP: "R: How do you feel when you're wearing your glasses (showing thumbs symbols)? P: Picks "thumbs up" symbol. R: Thumbs up. You like your glasses? P: Yeah." (INT_ID_IRL_001). Within the residential setting, all users felt included and received positive responses to the AP they were using. When users would go into the community, negative responses from others were sometimes experienced. For example, $R$ : What do they say? $P$ : They will be staring. $R$ : That's not really nice. P: No. Kids do it. (INT_ID_IRL_004). Overall, responses from others influenced people with ID to use or not use their AP. One of the professionals described it as: "P: I think the products help the person to be included in society, but society doesn't always include the product, if that makes sense". (INT_PRO_IRL_003). Use of AP was encouraged when the care system of the user was open to new AP. However, if carers or family were being reluctant to new AP, for example because of shame or fear, the use of AP was expected to be less. This is how one of the participants described it: "R: Do you sometimes see that people with an intellectual disability feel ashamed that they have to use the wheelchair or the product that you deliver? P: I don't think they feel ashamed, I think sometimes the family or the care network, it is usually the family, sometimes they will feel there is some kind of stigma attached to the use of the equipment or how they look". (INT_PRO_IRL_009). Overall, the stigma around ID seemed to be reduced compared to a few decades ago. And mainstream AP helped to reduce stigma around AP and facilitated using AP: "P: the preference would always be for something like an iPad, because it's mainstream, people have it, it's cool, it doesn't look different". (INT_PRO_IRL_003).

Customization. It was not always possible for people with ID to use mainstream AP and customization was often necessary. If customization was possible, it facilitated AP use for people with ID. Participants described the possibilities of both physical and cognitive customization options. If the customization process included different disciplines and experts, the outcomes were better fitted to the individual and facilitated continuous use. In some cases a multidisciplinary approach for customization was present: "P: But at the end of the day, like when you do apply that, so we say ok I want this, this, any possibilities to do this and then the vendor comes on board and the vendor will help us to do that. In most cases a physiotherapist actually jumps straight away in and we do modify that". (INT_PRO_IRL_004). If AP could not be customized to the individual needs, the use was not guaranteed. Participants described the difficulty to get AP customized for people with ID: "P: often it is difficult for them to access whether it's physical access or cognitively. But, no, I find that one of the areas of difficulty and some of the suppliers wouldn't really be able to adapt". (INT_PRO_IRL_002). A barrier of customization was that customized AP could not be trialed and it was unknown if the AP would be accepted and used by the individual.

Environment. Conditions outside the control of the individual played a major role for the person to use the AP. Examples given by participants were a lack of internet that was needed to use certain digital AP or the actual size of AP such as standing frames, being unfit for use in relevant environment frequented by users: "P: If someone is using it [iPad] for travel training, a lot of people won't have any data that they can use outside. So then you're kind of cut off from that". (INT_PRO_IRL_001).
Follow-up and maintenance. Follow-up and maintenance of AP were important factors of continuous use of AP. Standard followup for some of the AP, like spectacles, was carried out: " $R$ : And do you have to go back there, to the [optician]? To check if the glasses are still good for you? P: Every two years I have to go back. R: And they check it? P: And they check it again. They see what's, do you need a new pair or not". (INT_ID_IRL_002). For some individuals, the need for AP follow-up was discussed during the user's personal care plan (PCP) meeting. However, in most cases the carers would be the one to signal the need for follow-up or maintenance. In these situations carers could be a barrier or a facilitator; a barrier when they weren't competent to signal issues with the AP and its use, and a facilitator if they could: " $R$ : Do you sometimes see that, when you see a person again after six months, that there is something wrong with the equipment, but the person themselves or their people around them haven't noticed that there is something wrong with the product? P: Of course, a lot of incidents happen like that". (INT_PRO_IRL_004). Some of the users could signal the need for a review themselves: "R: And do you go back to her [physiotherapist] to check it, if it's still good for you? P: I told her about, when I'm holding it, your hand gets all red. R: Yeah when you're holding it, it can be very painful for the skin. P: She got me this rubber thing. R: Ok. That helped? P: It's helping". (INT_ID_IRL_003). Most of the suppliers would have a service to provide maintenance, but a waiting list may apply: " $P$ : she needs to get a new battery and a new charger. And we're waiting for another part for her chair. Waiting since the end of August and the battery came in, but there was another little part. It's the timeframe". (INT_ID_IRL_009).

Impact. If AP had a certain impact in the user's life and enabled activities for the user, it was expected that the user would continue using the AP: "P: We've had some really nice examples of where it has worked in, some of our adults of the adult service here actually went and volunteered at a local preschool where they were doing storytelling using their switches and their devices. That was really nice, it worked well for everyone, they loved meeting the children, and the children really anticipated their weekly visits really highly." (INT_PRO_IRL_002), or: "R: And does it [walker] help you to make friends? P: Yes. R: How? P: Because it helps me to get to the workshops. They aren't wheelchair friendly. $R$ : Ah, so if there's a workshop you want to go to and it's not wheelchair friendly you can take the walker? P: Yeah". (INT_ID_IRL_008).

Knowledge and awareness. Awareness of why AP was needed and understanding how to use the AP, were both facilitators for continuous use. Carers and users did need to be trained to know how to use the AP correctly: "P: Like staff would need quite a high level of support. You know, training how to use the device, because there is such a varied range of devices out there. Even sometimes I am even googling things before I meet them. But I suppose the staff would need a kind of high level of training. Not just about the device on itself, but also how to introduce the device appropriately, how to make sure it's going to be successful for the person". (INT_PRO_IRL_003). Understanding the purpose of AP was not always possible for people with a severe to profound level of ID. But they could learn cause and effect of the AP, which came down to familiarity. Some practical issues, like the huge staff turnover within ID care, hindered AP use in daily practice: "P: And it depends as well, sometimes it might be a phone call, sometimes it might be staff saying 'I have no idea, I have never heard of this piece of equipment in my life' and you're going 'what?'. But staff are a huge factor in all of that". (INT_PRO_IRL_003). It helped if individualized AP instructions were available to staff that 
explained how to help the person with ID use their AP. AP use was higher among persons who were able to independently use their AP, as stated by this participant: $R$ : And can you get into the wheelchair yourself? P: Self. P (carer): Yeah. R: And you put on the belt, do you do it yourself? P: Yeah. R: So nobody needs to help you with the wheelchair? You can do it all yourself? P: Yeah. R: That's very good. Also to start it? $P$ (carer): She can. Sometimes it's turned off at the back and she goes out and turn it on herself. So she is well able to. She charges it up every night herself. For the next day". (INT_ID_IRL_005). Some participants clearly explained they were able to use the AP themselves and how they remembered to use it. For example: "R: And do you put the insoles in your shoes yourself or does somebody help you? P: I do it myself. R: And do you sometimes forget? P: Sometimes I forget, they are calling me do this and do that. R: It's too busy and then you forget. Does the houseparent maybe sometimes remind you to put the insoles in your shoes? P: Sometimes. I'm good at thinking about it myself. I do my own washing. If I don't remember I put an $S$ on my hand". (INT_ID_IRL_003). One participant mentioned the possibilities of peer learning to increase use of AP: "P: there is say a group of people who are very used to using their iPhones for things and iPads and they are very savvy and then you have another group of people that might want to use those devices but might have other difficulties like you know, reading and things like that, so they can't access enough. And I've seen that the two groups kind of coming together and showing each other how to use things, which has been really nice, in the same group, they are in the same day center, but when you put people together it's like peer learning". (INT_PRO_IRL_001).

Support. Support played a huge role for persons with ID to (correctly) use their AP. They might need support because of cognitive limitations, physical disability or both: "R: And I suppose, the staff will use the phone for her, like put it to her ear. I don't think she can grab it herself? P: No, no. We put it on pillows, so we stand it near her and put it on loud speaker". (INT_ID_IRL_007). Time was an important factor for carers to provide AP support on a daily basis: "P: Generally the staff are very good about using them, but I suppose it's trying to get the, because they are trying to think of everything at one time, they are trying to think where the person has to go, what they need to do, so they are trying to think about everything. So it's often difficult to get them to actually use it [communication device] consistently". (INT_PRO_IRL_002). And carers or family needed to be convinced of the purpose and impact of the AP to be willing to provide that support: "P: But you know, sometimes working with families can be a bit of a battle like that, you have to really compromise and sometimes the person gets lost in that. Because the person might want to use the equipment but the family is not". (INT_PRO_IRL_001)

\section{APL suggestions}

The two main suggestions for the APL that were seen as essential for people with ID and which are missing on the current APL of the GATE program were hoists (and slings) and switches (an interface for individuals who have severe physical or cognitive disabilities that bypasses the need to do any complicated movement patterns), as mentioned by respectively six and three participants of group (2) Providers of AP $(n=15)$. Other suggestions mentioned by at least two participants were kitchen aids and adjusted cutlery, adjusted baths, dressing aids, environmental control systems (e.g., automatic door), and adjustable beds.

\section{Discussion}

This paper provides insights from the users' and providers' perspectives on factors influencing access and continues use of AP for people with ID. Within the context of this study, AP seems to be available close to the users and transport is well organized to get to the AP provider. Most of the health professionals working for ID service providers have the flexibility to assess the user in the user's own environment, or somewhere close to the user's home or daycare center, and they often involve the person with ID as much as possible during the assessment. These aspects are all facilitators to access AP that fits the users' needs. On the other hand, proactive assessment by health professionals is rare and the vast majority of the persons with ID depend on carers to signal the need for an assessment. This implies that carers need to be capable of identifying and acting on the needs of the person with ID. However, carers indicated a lack of knowledge and education around ID and AP. This may lead to a failure to recognize AP needs and with that, an underutilization of AP that could benefit the person with ID. For example, within our sample, no AP were currently used to support hearing and regular screening was not implemented. One participant abandoned her hearing aid due to problems in daily use, and the extent of follow-up by professionals was unclear. A large study in adults with ID showed that $30 \%$ of adults with ID have a hearing impairment and in half of the cases this was not known before the study took place [31]. For people with ID it is difficult to understand and express symptoms related to hearing impairment. In some cases it causes changes in behaviour which can be the only signal for carers.

A striking outcome for access to AP is the unequal funding system. Public funding was found to be segmented; different types of impairment may have different funding systems to acquire AP with funding dependant on both the number of people and the annual budget of the public funding authority within an area. Funding is known to be one of the main barriers to AP access for people with ID [22]. Fundamentally, the segmented and disjointed aspect of public funding found in this study is a key issue for policy makers who aspire to adhere to implementing the UNCRPD equally in all areas of their country. In addition, public funding in this study did not cover every type of AP and long waiting lists existed. Public funding authorities focussed on supporting AP that apply to comfort and basic needs, and less on AP that enables social inclusion. In other words, the public funding authority is still using the medical model of disability instead of the social model of disability and/ or the human rights model of disability which the UNCRPD codifies [32]. In relation to social inclusion, it is important to highlight that participants clearly stated a decline in stigma associated with ID and AP use for persons with ID. However, the paternalistic attitude of care providers towards people with ID meant a limited access and use of certain AP, such as mobile phones.

Another barrier to access AP that was found in this study was a lack of access to the mainstream workforce for people with ID. The mainstream workforce can act as an access point to technology, as one of the participants indicated. The current trend for governments is to shift from a centralized setting towards inclusion and community living, where people with ID would participate in mainstream services as much as possible, including workforces. The UNCRPD stipulates community living for people with ID; all people with disabilities should be part of the normal societal processes that include access to education, employment, and housing [33]. The Sustainable Development Goals (SDGs), aim to "leave no one behind" through inclusion of all people, including people with ID [34]. However, according to the outcomes of this study, people with ID do not have access to the mainstream workforce and AP is not sufficiently 
taken into account with the current shift to community living. AP has the potential to enhance independence and community living and use of AP should therefore, be encouraged to ensure fuller participation and inclusion of people with ID in society. With that, access points, follow-up and maintenance services of AP need to be available within community settings. In addition, health professionals in the community need to be trained to recognize and assess the different and specific needs of people with ID.

Abandonment of AP was not that common according to the participants of this study and did not seem a significant barrier to continuous use, which one would expect based on the literature [23]. One reason for this outcome could be that the participants included in this study were all living in group homes supported by an ID care service provider that facilitated AP use via onsite carers. Another reason could be that the health professionals only prescribed AP that really met the users' need. The health professionals were aware of the limitations of public funding, the waiting lists and the extensive administration process to get the AP, therefore they made sure the AP was trialed and accepted by the user before they ordered the AP.

People with ID hugely depend on carer's support to use their AP. However, due to high staff turnover in ID care, carers aren't always aware of the AP that is available or how it can best be used or adapted for persons with ID. It is important that carers and users are both aware of the purpose and impact of AP for the user and that they are trained how to use AP. This includes new carers to the service. Standard follow-up or maintenance of AP isn't always carried out, and so carers and users should also be aware when follow-up is needed. A pro-active approach from the providers of AP to conduct follow-up and consolidated maintenance is key and should always be considered for this population. It is clear that AP has to be provided systemically [35]; and this means thinking through the needs and opportunities for people with ID in terms of products [36], personnel [37], users [38], its provision [39] and policy [40]; as well as other systems issues.

Some contradictions between factors influencing access and factors influencing continuous use were found. Firstly, there didn't seem to be any problem in AP availability according to the participants, if the need was signalled and assessed. However, availability did not guarantee continuous use. If the environment didn't enable the use of AP consistently, such as internet availability across locations relevant to the user of digital AP, then the impact of the AP was reduced. Second, when people with ID could independently use (even if partially) their AP, continuous use was improved. The independent use of AP was observed as more often a possibility for people with mild to moderate ID.

There are a few limitations to this study. The main limitation is that persons with ID and prescribers of AP included in this study were linked to only one ID service provider (while several service providers are available in Ireland). This also resulted in solely female participants, as the locations of this ID service provider have a history of providing care primarily to females with ID. In addition, all persons with ID were living in a group home of the service provider, none of them were living with family. Although it was mentioned by participants that people with ID who live with family would also be linked in with the ID service provider and services, the care system is different and may influence access and use of AP. This means that the outcomes of this study cannot represent all situations for people with ID in Ireland or internationally. However, with relevance to existing literature, the situation does reflect the situation for many people with ID in the Irish context and key related developed territories. A limitation of the development of the interview guide was that the pilot testing was rather limited to only one person with mild ID and not to providers. However, the interview guide was vetted with colleagues, to solicit feedback on the scope, clarity and flow of questions, and where appropriate, the interview guide was refined. Another limitation was that the carers were asked to list the AP that were in current use by the person with ID. AP that wasn't individually owned by participants, for example, a hoist system within a group home, may not always have been listed. Numbers of AP currently in use as mentioned in the results section could, therefore be lower than the actual number. A fourth limitation is that the participants were not assessed for AP needs in the context of this study. So it remains unknown which participants with ID could need or benefit from AP but didn't have the relevant AP.

\section{Acknowledgements}

The authors would like to thank all staff and service users from the Daughters of Charity for their collaboration, and colleague John Owuor for his contribution.

\section{Disclosure statement}

No potential conflict of interest was reported by the authors.

\section{Data availability}

The data that support the findings of this study are available from the corresponding author, $\mathrm{FHB}$, upon reasonable request.

\section{Funding}

This research was supported by funding from the charity RESPECT and the People Programme (Marie Curie Actions) of the European Union's Seventh Framework Programme (FP7/2007-2013) under http://dx.doi.org/10.13039/501100000783 REA grant agreement no. PCOFUND-GA-2013-608728.

\section{References}

[1] WHO. Global Cooperation on Assistive Technology (GATE) 2014 [cited 2018 July 30]. Available from: www.who.int/ phi/implementation/assistive_technology/phi_gate

[2] Tebbutt E, Brodmann R, Borg J, et al. Assistive products and the Sustainable Development Goals (SDGs). Global Health. 2016;12:79.

[3] WHO. Priority Assistive Products List (APL) 2016 [cited 2016 28 September]; Priority Assistive Products List (APL)]. Available from: http://www.who.int/phi/implementation/ assistive_technology/global_survey-apl/en/

[4] Owuor J, Larkan F, MacLachlan M. Leaving no-one behind: using assistive technology to enhance community living for people with intellectual disability. Disabil Rehabil Assist Technol. 2017;12:426-428.

[5] Bryant BR, Seok S, Ok M, et al. Individuals with intellectual and/or developmental disabilities use of assistive technology devices in support provision. J Special Edu Technol. 2012;27: 41-57. PubMed PMID: 1361841558; EJ1001424; English.

[6] Carmeli E, Imam B, Merrick J. Assistive technology and older adults. I.L. Rubin et al, editors. Health Care for People with Intellectual and Developmental Disabilities across the Lifespan. Springer International Publishing Switzerland; 2016. p. 1465-1471. 
[7] Lewis RB, Lewis RB. Assistive technology and learning disabilities: today's realities and tomorrow's promises. J Learn Disabil. 1998;31:16-26. PubMed PMID: 9455174; eng.

[8] AAIDD. Definition of Intellectual Disability 2013 [cited 2016 Oct 05]. Available from: https://aaidd.org/intellectual-disability/definition\#.V_UMUIWcHIU

[9] WHO. ICD-10 Mental Retardation 2016 [cited 2016 Oct 03]; ICD-10 mental retardation]. Available from: http://apps.who. int/classifications/icd10/browse/2016/en\#/F70-F79

[10] Association AP. Diagnostic and statistical manual of mental disorders-DSM-5. 5 ed. American Psychiatric Publishing. Diagnostic and Statistical Manual of Mental Disorders - DSM-5. 5th edition: American Psychiatric Publishing; Washington; 2013.

[11] Hatton C, Emerson E. International review of research in developmental disabilities - health disparities and intellectual disabilities. Vol. 48. Academic Press Elsevier; 2015.

[12] Haveman M, Perry J, Salvador-Carulla L, et al. Ageing and health status in adults with intellectual disabilities: results of the European POMONA II study. J Intellect Dev Disabil. 2011;36:49-60.

[13] Jansen A, Kingma-Thijsen J. Searching for physical explanations for challenging behaviour in people with an intellectual disability (in Dutch). Centrum voor Consultatie en Expertise. Utrecht, The Netherlands; 2011:191.

[14] Patja $K$, livanainen $M$, Vesala $H$, et al. Life expectancy of people with intellectual disability: a 35-year follow-up study. J Intellect Disabil Res. 2000;44:591-599.

[15] Wehmeyer ML. The use of assistive technology by people with mental retardation and barriers to this outcome: a pilot study. Technol Disabil. 1995;4:195-204. PubMed PMID: WOS:A1995TN62400003.

[16] Kaye HS, Yeager P, Reed M. Disparities in usage of assistive technology among people with disabilities. Assist Technol. 2008;20:194-203. PubMed PMID: WOS:000261938400002.

[17] Carey AC, Friedman MG, Bryen DN. Use of electronic technologies by people with intellectual disabilities. Mental Retard. 2005;43:322-333. PubMed PMID: 16131229; eng.

[18] Baxter $\mathrm{H}$, Lowe $\mathrm{K}$, Houston $\mathrm{H}$, et al. Previously unidentified morbidity in patients with intellectual disability. $\mathrm{Br} \mathrm{J}$ Gen Pract. 2006;56:93-98. PubMed PMID: PMC1828252.

[19] WHO. Ageing and intellectual disabilities - improving longevity and promoting healthy ageing: summative report. Geneva, Switzerland: World Health Organization; 2000. [cited 2017 Oct 06]. Available from: http://www.who.int/ mental_health/media/en/20.pdf

[20] Houses of the Oireachtas. Committee on the Future of Healthcare 2017. Available from: http://www.oireachtas.ie/ parliament/media/committees/futureofhealthcare/SlaintecareReport-May-2017.pdf last accessed data: 04 December 2018

[21] WHO. International classification of functioning, disability, and health: ICF. Geneva. 2001. Available from: www.who.int/ classifications/icf/en/. Last accessed data: 04 December 2018

[22] Boot FH, Owuor J, Dinsmore J, et al. Access to assistive technology for people with intellectual disabilities: a systematic review to identify barriers and facilitators. J Intellect Disabil Res. 2018;62:900-921. doi: 10.1111/jir.12532.

[23] Scherer MJ. From people-centered to person-centered services, and back again. Disabil Rehabil Assist Technol. 2014;9: $1-2$.

[24] Federici S, Meloni F, Borsci S. The abandonment of assistive technology in Italy: a survey of National Health Service users. Eur J Phys Rehabil Med. 2016;52:516-526.
[25] Scherer MJ, Federici S. Why people use and don't use technologies: introduction to the special issue on assistive technologies for cognition/cognitive support technologies. Nre. 2015;37:315-319. PubMed PMID: 26518529; eng.

[26] Brodin J. Implementation of new technology for persons with mental retardation and the importance of staff education. Int J Rehabil Res. 1998;21:155-168. PubMed PMID: 9924678; eng.

[27] Palmer SB, Wehmeyer ML, Davies DK, et al. Family members' reports of the technology use of family members with intellectual and developmental disabilities. J Intellect Disabil Res. 2012;56:402-414. PubMed PMID: 21988242; eng.

[28] van Schijndel-Speet $M$, Evenhuis $H M$, van Wijck $R$, et al. Facilitators and barriers to physical activity as perceived by older adults with intellectual disability. Intellect Dev Disabil. 2014;52:175-186.

[29] IRN. Inclusive Research Network 2008 [Sept 2018]. Available from: http://www.fedvol.ie/Inclusive_Research_Network_ IRN/Default.241.html

[30] Elliott R, Timulak L. Descriptive and interpretive approaches to qualitative research. In: Miles J, Gilbert $\mathrm{P}$, editors. A handbook of research methods for clinical and health psychology. New York, NY: Oxford University Press; 2005. p. 147-160.

[31] Meuwese-Jongejeugd A, Vink $M$, van Zanten $B$, et al. Prevalence of hearing loss in 1598 adults with an intellectual disability: cross-sectional population based study. Int J Audiol. 2006;45:660-669. PubMed PMID: 17118908; eng.

[32] Degener T. Disability in a human rights context. Laws. 2016;5:35. PubMed PMID

[33] UN. Convention on the Rights of Persons with Disabilities (CRPD) 2006 [cited 2017 Sept 18]. Available from: https:// www.un.org/development/desa/disabilities/convention-onthe-rights-of-persons-with-disabilities.html

[34] UN. Sustainable Development Goals 2015 [cited Dec 2017]. Available from: http://www.un.org/sustainabledevelopment/sustainable-development-goals/

[35] MacLachlan M, Scherer M. Systems thinking for assistive technology: a commentary on the GREAT summit. Disabil Rehabil Assist Technol. 2018;13:492-496.

[36] Smith RO, Scherer M, Cooper R, et al. Assistive technology products: a position paper from the first global research, innovation, and education on assistive technology (GREAT) summit. Disabil Rehabil Assist Technol. 2018;13:473-485.

[37] Smith EM, Gowran RJ, Mannan H, et al. Enabling appropriate personnel skill-mix for progressive realization of equitable access to assistive technology. Disabil Rehabil Assist Technol. 2018;13:445-453.

[38] Desmond D, Layton N, Bentley J, et al. Assistive technology and people: a position paper from the first global research, innovation and education on assistive technology (GREAT) summit. Disabil Rehabil Assist Technol. 2018;13:437-444.

[39] de Witte L, Steel E, Gupta S, et al. Assistive technology provision: towards an international framework for assuring availability and accessibility of affordable high-quality assistive technology. Disabil Rehabil Assist Technol. 2018; 13:467-472.

[40] MacLachlan M, Banes D, Bell D, et al. Assistive technology policy: a position paper from the first global research, innovation, and education on assistive technology (GREAT) summit. Disabil Rehabil Assist Technol. 2018;13:454-466. 